Research Paper

\title{
Expression of WDR79 is associated with TP53 mutation and poor prognosis in surgically resected non-small cell lung cancer
}

\author{
Jinwu Peng1,3,4, Yuting Zhan², Juan Feng22, Songqing Fan ${ }^{2}$ and Hongjing Zang ${ }^{\circledR}$ \\ 1. Department of Pathology, Xiangya Basic Medical School, Central South University, Changsha 410013, Hunan, China \\ 2. Department of Pathology, The Second Xiangya Hospital, Central South University, Changsha, 410011, Hunan, China \\ 3. Department of Pathology, Xiangya Changde Hospital, Changde 415000, Hunan, China \\ 4. Department of Pathology, Xiangya Hospital, Central South University, Changsha 410008, Hunan, China \\ $\triangle$ Corresponding authors: Songqing Fan, Department of Pathology, The Second Xiangya Hospital, Central South University, Changsha, Hunan, 410011, China. \\ E-mail address: songqingfan@csu.edu.cn. Hongjing Zang is a co-corresponding author of this paper with Songqing Fan. Her email address is \\ zanghongjing1991@163.com \\ (c) Ivyspring International Publisher. This is an open access article distributed under the terms of the Creative Commons Attribution (CC BY-NC) license \\ (https://creativecommons.org/licenses/by-nc/4.0/). See http://ivyspring.com/terms for full terms and conditions.
}

Received: 2018.10.11; Accepted: 2019.04.30; Published: 2019.06 .02

\begin{abstract}
Non-small cell lung cancer (NSCLC) represents a major health burden globally. WD repeat protein 79 (WDR79) is a member of the WD-repeat protein family. WDR79 is a highly conserved and natural antisense transcript to TP53 gene and involved in carcinogenesis of various types of cancer. Whether the alterations of WDR79 protein expression are associated with TP53 mutation and clinicopathological and prognostic implications in the patients with surgically resected NSCLC have not been reported. The purposes of the present study are to investigate the association between the expression of WDR79 and mutant p53 (mtp53) and clinicopathological features in NSCLC by immunohistochemistry. The results showed that positive expression of WDR79 $(58.8 \%, 170 / 289)$ and mtp53 $(48.1 \%, 139 / 289)$ in NSCLC was significantly higher than that in non-cancerous control lung tissues $(5.7 \%, 3 / 53$ and $1.9 \%, 1 / 53$, respectively). There was a significantly higher positive percentage of WDR79 expression in NSCLC with lymph node metastasis. The statistically positive correlation between WDR79 and mtp53 expression $(r=0.212$, $P=0.014)$ was identified by Spearman's rank correlation analysis. Kaplan-Meier survival curve analysis indicated that positive expression of WDR79 and common positive expression of WDR79 and mtp53 were correlated with poor overall survival rates in NSCLC patients $(P=0.029$ and $P=$ 0.041, respectively). Multivariate Cox regression analysis further identified that WDR79 positive expression was an independent unfavorable prognostic factor of NSCLC $(P=0.034)$. Taken together, positive expression of WDR79 proteins may be related with TP53 mutations and act as valuable independent biomarker to predict poor prognosis of patients with surgically resected NSCLC.
\end{abstract}

Key words: Non-small cell lung cancer, WDR79, TP53, mutant, prognosis

\section{Introduction}

Lung cancer is the leading cause of cancer-related deaths worldwide [1]. Lung cancer is classified into non-small cell lung cancer (NSCLC) and small cell lung cancer (SCLC) by pathological features. NSCLC accounts for approximately $80 \%$ of lung cancers. Although current therapies have considerably improved and an increasing number of new molecular targeted agents have been cleared for use in the treatment of NSCLC patients ${ }^{[2]}$, and the overall 5-year survival rate in advanced NSCLC is still less than $18 \%{ }^{[3]}$. Therefore, it is an urgent requirement to find new therapeutic targets for the treatment of NSCLC. TP53 gene is a famous tumor-suppressor gene and p53 alterations occurs in 
many epithelial carcinomas [4]. TP53 gene mutations are studied extensively, commonly recognized as related to carcinogenesis ${ }^{[5]}$. TP53 gene mutations also frequently occurred in NSCLC [6-8, 31]. The accurate conventional method for detecting TP53 mutations is next-generation sequencing (NGS) which costs much and takes a long time. In fact, NGS technique claims specific laboratory and instruments and trained pathologists, which limit the application and popularity of it. Because of easier operation and low cost, Immunohistochemistry (IHC) is wildly used in clinical diagnoses and translational tissue-based research. Disappointingly, half-life of wild-type p53 protein is too short to be detected by IHC, and IHC positive p53 may relate to some types of p53 mutations ${ }^{[9]}$, though different antibodies seem no significant difference in IHC method for NSCLC [10]. Intriguingly, Yemelyanova et al. found that p53 IHC staining was related with TP53 mutations or wild-type p53 to some extent in ovarian carcinoma [9].

WD-repeat protein 79 (WDR79), also known as WRAP53 and TCAB1, is a highly conserved and natural antisense transcript to TP53 gene [11]. What's more, WDR79 can regulate $\mathrm{p} 53$ protein expression via targeting p53 mRNA[12], and WDR79 gene is regarded as a bidirectional gene partner to TP53 [13]. Sun et al. found that overexpression of WDR79 is related with tumor progression in NSCLC [14]. Another research in head and neck carcinomas demonstrated that WDR79 may accelerate the tumor occurrence and development [15]. However, whether expression of WDR79 and mtp53 proteins is associated with clinicopathological features and prognostic implications in NSCLC has not been reported.

In this study, we detected the expression of WDR79 and mtp53 proteins by IHC in 289 cases of surgically resected NSCLC and 53 cases of non-cancerous lung control tissue, and investigated the correlations between the expression of WDR79 and mtp53 and clinicopathological features and prognostic implications in NSCLC.

\section{Materials and methods}

\subsection{Ethics statement}

All samples were obtained with informed consent, and all protocols, specimen usage, and data retrieval were approved by the Ethics Review Committee of the Second Xiangya Hospital of Central South University (Scientific and Research Ethics Committee, No. S039/2011). Written informed consent was obtained from all patients, also the written informed consent was obtained from the next of kin, caretakers, or guardians on the behalf of the minors/children participants involved in our study.

\subsection{Clinical data}

In this study, two hundred and eighty-nine (289) cases of paraffin-embedded NSCLC from the primary NSCLC patients with their age ranging from 41 to 71 years (average 55.84 years), also 53 cases of non-cancerous lung control specimen from independent patients with non-cancerous pulmonary diseases were collected from the Department of Pathology, The Second Xiangya Hospital of Central South University (Changsha, China) during the period from 2010 to 2016. NSCLC patients had undergone clinical surgery at the Department of Thoracic Surgery at the Second Xiangya Hospital of Central South University. All patients with NSCLC had been assessed and staged routinely and accepted definitive surgical resection of the lung and systematic mediastinal lymph node dissection. No patients had been previously treated with radiotherapy or chemotherapy before operation. Patients had a confirmed histological diagnosis of NSCLC according to WHO histological classification of the lung cancer. The staging classification of the current analysis was carried out based on the criteria of the 7th edition of the AJCC/UICC TNM staging system of lung cancer (2009). Clinicopathological features of patients with NSCLC and non-cancerous control lung tissues were shown on Table 1. This study was carried out under the approval and supervision of the Ethics Committee of The Second Xiangya Hospital. Complete clinical record and followed-up data were available for all patients. In this study, we used the tissue microarrays (TMA) technology designed and constructed high-throughput NSCLC TMAs according to the technical rules previously described [16,17].

\subsection{IHC staining and scores}

The IHC for the detection of WDR79 and mtp53 expression in NSCLC TMAs was carried out using ready-to-use Envision $\mathrm{TM}^{+}$Dual Link System-HRP methods (Dako; Carpintrria, CA). As described in detail previously [16, 18], in briefly, each section was deparaffinized and rehydrated, and citrate-mediated high-temperature microwave antigen retrieval was performed for 15 minutes. After cooled down to room temperature, the samples were immersed into methanol containing $3 \% \quad \mathrm{H}_{2} \mathrm{O}_{2}$ to inactivate endogenous peroxidase at room temperature for 30 minutes. To eliminate nonspecific staining, the slides were incubated with appropriate preimmune serum for 30 minutes at room temperature, and then incubating with specific first antibody in $4^{\circ} \mathrm{C}$ overnight in wet box. The staining conditions for each antibody were adjusted according to our laboratory experience, 1:100 dilution of primary antibody to 
WDR79 protein (Bethyl Laboratories, Inc., Montgomery, TX, USA), with a 1:100 dilution of primary anti-mtp53 antibody [E26] (Rabbit monoclonal, ab32389, Abcam, Cambridge, UK). The second antibody conjugated with a labeled polymer-HRP was added according to the manufacturer's instructions and incubated at room temperatures for 30 minutes. Color reaction was developed by $\mathrm{DAB}$ (3, 3-diaminobenzidine) chromogen solution. All slides were counterstained with hematoxylin. Positive control slides were included in every experiment in addition to the internal positive control. The specificity of the antibody was determined with matched IgG isotype antibody as a negative control.

Table 1. Clinicopathological features of patients with NSCLC and non-cancerous control lung tissues

\begin{tabular}{ll}
\hline Variable & No. of patients (\%) \\
\hline Age(y) & $75(26.0)$ \\
$\leq 50$ & $214(74.0)$ \\
$>50$ & $217(75.1)$ \\
Gender & $72(24.9)$ \\
Male & \\
Female & $74(25.6)$ \\
Clinical stages & $67(23.2)$ \\
Stage I & $148(51.2)$ \\
Stage II & \\
Stage III & $125(43.3)$ \\
Lymph node status & $164(56.7)$ \\
N0 & \\
N1/N2/N3 & $144(49.8)$ \\
Histological types & $145(50.2)$ \\
SCC & \\
ADC & $4(1.4)$ \\
Pathological grades & $127(43.9)$ \\
Well & $158(54.7)$ \\
Moderate & \\
Poor & $124(42.9)$ \\
Smoking status & $165(57.1)$ \\
Smoker & \\
Non-smoker & $179(61.9)$ \\
Survival status & $110(38.1)$ \\
Alive & \\
Dead & $17(32.1)$ \\
Non-cancerous lung tissues & \\
Age(y) & \\
$\leq 50$ & $36(67.9)$ \\
$>50$ & $37(69.8)$ \\
Gender & $16(30.2)$ \\
Male & \\
Female & $20(37.7)$ \\
Smoking status & $33(62.3)$ \\
Smoker & \\
Non-smoker & \\
\hline Abbreviation: SCC: squamous cell carcinoma: ADC: adenocarcinoma. \\
\end{tabular}

Abbreviation: SCC: squamous cell carcinoma; ADC: adenocarcinoma

Immunohistochemical staining was evaluated independently by SF and JP who were blinded to the clinicopathological data, at $200 \times$ magnification light microscopy. Positive staining of WDR79 protein mainly located in both the nuclei and cytoplasm of cancer cells. Positive expression of mtp53protein was found in the nuclei.
A semiquantitative evaluation of WDR79 protein expression was performed using a method described as follows [16]: Staining intensity for WDR79 was divided into four grades (intensity scores): as 0 (negative, no staining), 1 (weak, light brown), 2 (moderate, brown), and 3 (strong, dark brown). The positive percentage was divided into five grades (percentage scores): $0(0 \%), 1(1 \%-25 \%), 2(26 \%-50 \%), 3$ $(51 \%-75 \%)$, and $4(76 \%-100 \%)$. Staining positivity was determined by the formula: overall scores = percentage score $\times$ intensity score. The result of the staining scores was used as the final staining score for WDR79 (0-12). Its optimal cut off score was 4, which was on the basis of the overall survival of NSCLC patients using the log-rank test. A score of $\geq 4$ was considered a positive expression of WDR79 while scores less than 4 were considered negative expression of WDR79.

p53 IHC scoring system was performed using a method described in the literature ${ }^{[19]}$ as follows: Normal or wild type pattern is characterized by variable staining intensity, the slides were scored as negative expression of mtp53 protein. Mutant type p53 is characterized by both strongly intense staining in all cancer cell nuclei and complete absence of expression within cell nuclei, the slides were scored as positive expression of mtp53 protein. Agreement between the two evaluators was $95 \%$, and all scoring discrepancies were resolved through discussion between the two evaluators under a two-headed microscope.

\subsection{Statistical analysis}

All statistical analyses were performed using SPSS version 20.0 (SPSS, Chicago, IL). The Chi-square test was used to analyze the relationship between expression of WDR79 and mtp53 and clinicopathological characteristics of NSCLC. Spearman rank correlation coefficient was used to assess the significance of the association among expression of WDR79 and mtp53 in NSCLC. Kaplan-Meier analysis was performed to obtain overall survival curves, and statistical significance was assessed using the log-rank test. Overall survival was defined as the time from the treatment initiation (diagnosis) to the date of death or date of last follow-up. To identify whether positive expression of WDR79 and mtp53 was the independent prognostic factor for overall survival in NSCLC, multivariate analysis using the Cox proportional hazard regression model was performed. All $P$-values were based on 2 -sided statistical analysis, and $P<0.05$ was considered statistically significant. 


\section{Results}

\subsection{Expression of WDR79 and mtp53 was significantly higher in NSCLC}

We separately detected positive expression and cellular localization of WDR79 and mtp53 proteins in 289 cases of NSCLC tissues and 53 cases of non-cancerous control lung tissues. WDR79 protein was found positive expression in both cytoplasm and nuclear of lung SCC and lung ADC (Figure 1A and Figure 1B), also positive expression in the non-cancerous control lung bronchial epithelia (Figure 1C). While mtp53 was only expressed in nuclear of lung SCC and lung ADC (Figure 1D and Figure 1E). The negative control was no staining of mtp53 protein in the lung SCC (Figure 1F). Furthermore, the positive percentage of WDR79 and mtp53 expression in the NSCLC and non-cancerous control lung tissues was 58.8\% (170/289) and $48.1 \%$ (139/289), 5.7\% (3/53) and 1.9\% (1/53), respectively (Figure 2). The results demonstrated that positive percentages of WDR79 and mtp53 expression were significantly increased in NSCLC tissues compared to non-cancerous control lung tissues $(P<0.001$ and $P<0.001$, respectively). Moreover, the association between positive expression of WDR79 and mtp53 proteins in 289 cases of NSCLC was investigated by Spearman's rank correlation analysis. Among 289 cases of NSCLC cases, 87 patients were found to express WDR79 and mtp53 commonly. There was statistically positive correlation between WDR79 and mtp53 expression $(\mathrm{r}=0.212, P=0.014)$.

\subsection{Associations between expression of WDR79 and mtp53 proteins and clinicopathological characteristics of NSCLC}

We further investigated the associations between the positive expression of WDR79 and mtp53 and clinicalpathological characteristics of NSCLC including age, gender, smoking status, histological type, clinical stage and lymph nodes metastasis (LNM) in univariate chi-square test. Data were shown in Table 2. For WDR79 individually, positive percentages of WDR79 expression in NSCLC with LNM $(64.0 \%)$ was significantly higher than that in patients without LNM $(52.0 \%) \quad(P=0.040)$, which indicated that WDR79 may be related with NSCLC progression and poor prognosis. As for mtp53 protein expression individually, the positive percentages of mtp53 were evidently higher in the senior-age group NSCLC patients $(51.9 \%)$ than in junior group (37.3\%) $(P=0.030)$. Also, the expression of WDR79 and mtp53 protein had a positive correlation with smoking $(P=0.015$ and 0.007 respectively). Moreover, positive percentages of $\mathrm{mtp} 53$ protein expression in the lung SCC $(54.9 \%)$ were significantly higher than that in the lung $\operatorname{ADC}(41.4 \%)(P=0.022)$.
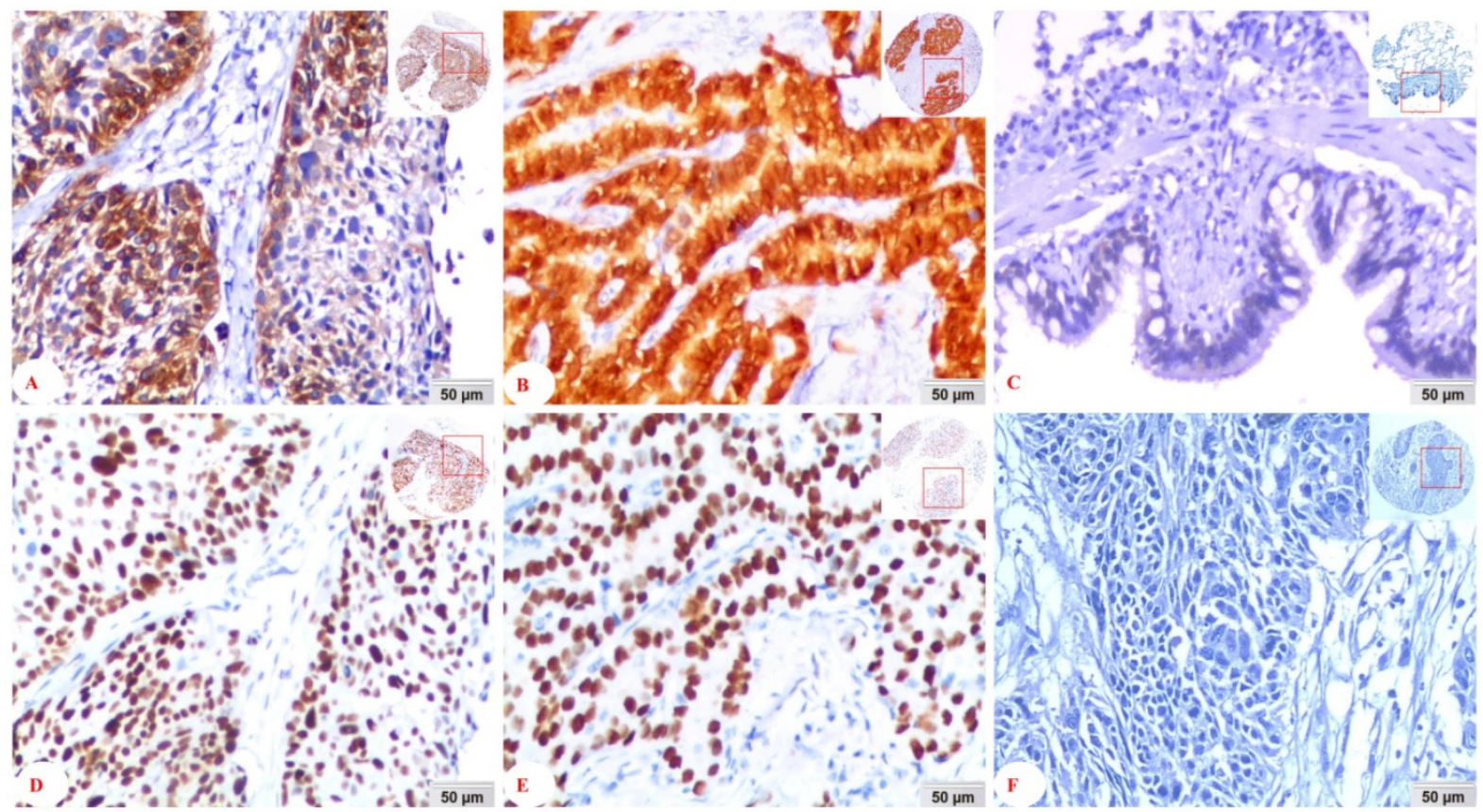

Figure 1. Expression of WDR79 and mtp53 proteins in NSCLC detected by IHC. A: WDR79 positive expression in the cytoplasm and nuclear of lung SCC; B: WDR79 positive expression in the cytoplasm and nuclear of lung ADC; C: WDR79 positive expression in the cytoplasm and nuclear of bronchial epithelia of non-cancerous control normal lung tissues; D: mtp53 positive expression was located in the nuclear of lung SCC, F: mtp53 positive expression in the nuclear of lung ADC; G: The negative control was no staining of mtp 53 protein in the lung SCC (IHC, DAB staining, 20x magnification). 


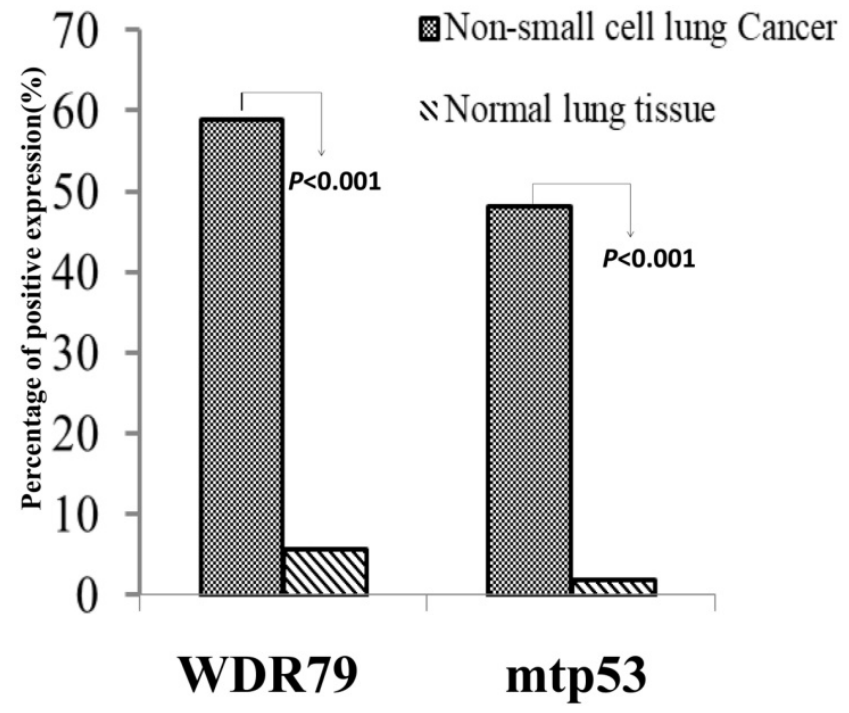

Figure 2. Positive expression of WDR79 and mtp53 in NSCLC and non-cancerous control normal lung tissues was compared by Chi-square test. Column charts showed that there were significant differences between the groups which were statistically evaluated by Chi-square test. Positive expression of WDR79 and mtp53 proteins was evidently higher in the NSCLC compared to the non-cancerous control normal lung tissues $(P<0.001$ and $P<0.001$, respectively).

Taken expression of WDR79 and mtp53 both in consideration, common positive expression of WDR79 and mtp53 was less occurred in the lung ADC (24.8\%) compared to the lung SCC $(35.4 \%)(P=0.05)$, which is a marginal value as shown in Table 2 . Besides, it was found common positive expression rate of WDR79 and mtp53 was higher in smoking patients $(P=0.025)$. No correlation was found among common expression of WDR79 and mtp53 and the other clinical pathology parameters, such as age, gender, clinical stages, LNM status and pathological grades $(P>0.05)$. In conclusion, overexpression of WDR79 may promote lymph node metastasis and poor prognosis of NSCLC.

\subsection{Impact of positive expression of WDR79 and mtp53 on overall survival rates of patients with NSCLC}

Kaplan-Meier curves for overall survival (OS) rates in NSCLC were analyzed (Figure 3). Positive WDR79 expression was related with shorter OS rates of NSCLC patients $(P=0.029)$ (Figure 3A). Also, mtp53 positive expression had significantly inverse relevance with OS rates, either as common positive expression of mtp53 and WDR79 $(P=0.026$ and $P=0.041$, respectively) (Figure $3 \mathrm{~B}$ and Figure 3C). In addition, NSCLC patients with poor pathological grades had worse overall survival rates than that of NSCLC patients with well/moderate pathological grades $(P=0.001)$ (Figure 3D). Meanwhile, Kaplan-Meier curves showed worse overall survival rates for NSCLC patients with lymph node metastasis compared to patients without lymph node metastasis $(P<0.001)$ (Figure 3E). As expected, NSCLC patients with early clinical stages (clinical stage I and stage II) had significantly longer OS times than the patients with late clinical stages (clinical stage III) $(P<0.001)$ (Figure 3F).

Table 2. Analysis of the association between expression of WDR79 and mtp53 proteins and clinicopathological features of NSCLC $(n=289)$

\begin{tabular}{|c|c|c|c|c|c|c|c|c|c|}
\hline \multirow[t]{2}{*}{ Clinicopathological features (n) } & \multicolumn{3}{|l|}{ WDR79 } & \multicolumn{3}{|l|}{ mtp53 } & \multicolumn{3}{|c|}{ WDR79/mtp53\# } \\
\hline & $\mathrm{P}(\%)$ & $\mathrm{N}(\%)$ & $P$-Value & $\mathrm{P}(\%)$ & $\mathrm{N}(\%)$ & $P$-Value & $\mathrm{P}(\%)$ & $\mathrm{N}(\%)$ & $P$-Value \\
\hline \multicolumn{10}{|l|}{ Age(years) } \\
\hline$\leq 50(n=75)$ & $41(54.7)$ & $34(45.3)$ & & $28(37.3)$ & $47(62.7)$ & & $21(28.0)$ & $54(72.0)$ & \\
\hline$>50(n=214)$ & $129(60.3)$ & $85(39.7)$ & 0.395 & $111(51.9)$ & $103(48.1)$ & 0.030 & $66(30.8)$ & $148(69.2)$ & 0.644 \\
\hline \multicolumn{10}{|l|}{ Gender } \\
\hline Male(n=217) & $121(55.8)$ & $96(44.2)$ & & $108(49.8)$ & $109(50.2)$ & & $67(30.9)$ & $150(69.1)$ & \\
\hline Female $(\mathrm{n}=72)$ & $49(68.1)$ & $23(31.9)$ & 0.066 & $31(43.1)$ & $41(56.9)$ & 0.323 & $20(27.8)$ & $52(72.2)$ & 0.620 \\
\hline \multicolumn{10}{|l|}{ Smoking status } \\
\hline Smoker (124) & $83(66.9)$ & $41(33.1)$ & & $71(57.3)$ & $53(42.7)$ & & $46(37.1)$ & $78(62.9)$ & \\
\hline Non-smoker (165) & $87(52.7)$ & $78(47.3)$ & $0.015^{*}$ & $68(41.2)$ & $97(58.8)$ & $0.007^{*}$ & $41(24.8)$ & $124(75.2)$ & $0.025^{*}$ \\
\hline \multicolumn{10}{|l|}{ Clinical stages } \\
\hline Stage I and II $(\mathrm{n}=141)$ & $80(56.7)$ & $61(43.3)$ & & 72(51.1) & $69(48.9)$ & & $40(28.4)$ & 101(71.6) & \\
\hline Stage III ( $\mathrm{n}=148)$ & $90(60.8)$ & $58(39.2)$ & 0.482 & $67(45.3)$ & $81(54.7)$ & 0.324 & $47(31.8)$ & $101(68.2)$ & 0.530 \\
\hline \multicolumn{10}{|l|}{ LNM status } \\
\hline $\operatorname{LNM}(n=164)$ & $105(64.0)$ & $59(36.0)$ & & $81(49.4)$ & $83(50.6)$ & & $53(32.3)$ & $111(67.7)$ & \\
\hline No LNM $(n=125)$ & $65(52.0)$ & $60(48.0)$ & $0.040^{*}$ & $58(46.4)$ & $67(53.6)$ & 0.614 & $34(27.2)$ & $91(72.8)$ & 0.347 \\
\hline \multicolumn{10}{|l|}{ Histological type } \\
\hline $\operatorname{SCC}(n=144)$ & $89(61.8)$ & $55(38.2)$ & & $79(54.9)$ & $65(45.1)$ & & $51(35.4)$ & $93(64.6)$ & \\
\hline $\operatorname{ADC}(n=145)$ & $81(55.9)$ & $64(44.1)$ & 0.345 & $60(41.4)$ & $85(58.6)$ & 0.022 & $36(24.8)$ & $109(75.2)$ & 0.050 \\
\hline \multicolumn{10}{|l|}{ Pathological grades } \\
\hline Well/Moderate $(\mathrm{n}=131)$ & $80(61.1)$ & $51(38.9)$ & & $56(42.7)$ & $75(57.3)$ & & $41(31.3)$ & $90(68.7)$ & \\
\hline Poor $(n=158)$ & $90(57.0)$ & $68(43.0)$ & 0.480 & $83(52.5)$ & $75(47.5)$ & 0.097 & $46(29.1)$ & $112(70.9)$ & 0.687 \\
\hline
\end{tabular}

Abbreviation: LNM, lymph node metastasis; SCC: squamous cell carcinoma; ADC: adenocarcinoma; P: positive; N: negative.

Note: WDR79/mtp53": P was common positive expression of WDR79 and mtp53; N was all other immunophenotype of WDR79 and mtp53 expression; *Chi-square test was used. $P<0.05$ was regarded as statistically significant. 

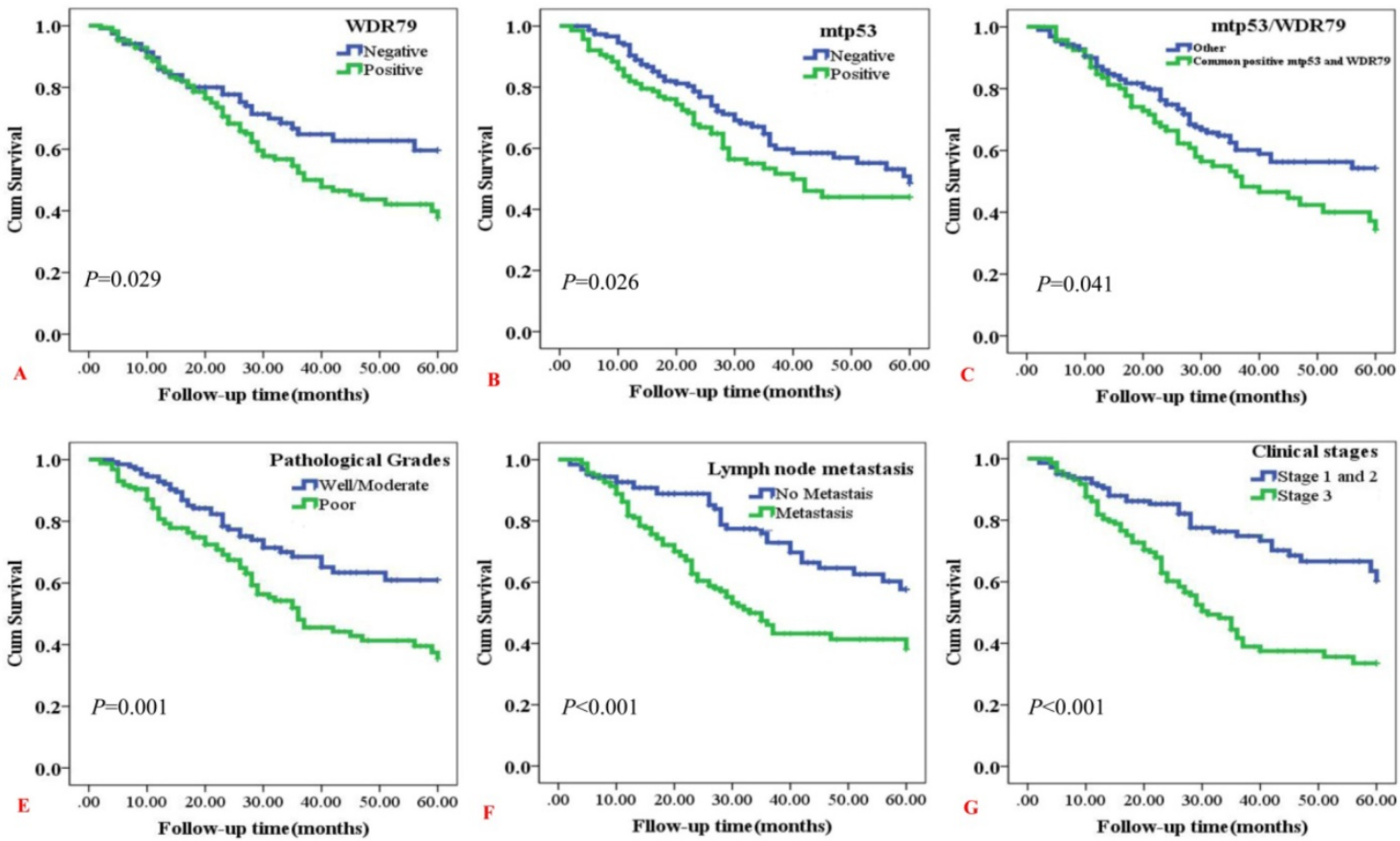

Figure 3. Kaplan-Meier overall survival curves of NSCLC patients with expression of WDR79 and $\mathrm{mtp} 53$ proteins and clinicopathological characteristics were statistically evaluated by the log-rank test. A: Positive expression of WDR79 had significantly correlation with worse overall survival (OS) rates of NSCLC patients $(P=0.029$, two sided). B: Kaplan-Meier curves showed worse OS rates for NSCLC patients with positive expression of mtp53 protein compared to patients with mtp53 negative staining $(P=0.026$, two sided). C: Kaplan-Meier curves showed worse OS rates for NSCLC patients with common expression of WDR79 and mtp53 compared to other immunophenotypes of WDR79 and mtp53 ( $P=0.041$, two sided). D: Kaplan-Meier curves showed worse OS rates for NSCLC patients with poor pathological grades compared to patients with well/moderate grades $(P<0.001$, two sided). E: Kaplan-Meier curves showed worse OS rates for NSCLC patients with lymph node metastasis compared to patients without lymph node metastasis $(P<0.001$, two sided). F: Kaplan-Meier curves showed worse OS rates for NSCLC patients with clinical stage III compared to patients with clinical stage I and II $(P<0.001$, two sided).

Table 3. Summary of Multivariate Cox Regression Analysis Results of Overall Survival

\begin{tabular}{llllllll}
\hline Parameter & B & S.E. & Wald & $P$-value & HR & \multicolumn{2}{c}{ 95.0\%CI for HR } \\
\cline { 7 - 9 } & & & & & & Lower & Upper \\
\hline Age & 0.220 & 0.233 & 0.895 & 0.344 & 1.246 & 0.790 & 1.966 \\
Gender & -0.520 & .252 & 4.264 & $0.039^{*}$ & 0.595 & 0.363 & 0.974 \\
Smoking status & 0.311 & .231 & 1.386 & 0.549 & 1.302 & 0.549 & 3.086 \\
Histological types & 0.230 & .210 & 1.204 & 0.272 & 1.259 & .834 & 1.900 \\
LNM status & 0.494 & 0.235 & 4.417 & $0.036^{*}$ & 1.638 & 1.034 & 2.596 \\
Treatment strategy & -0.002 & 0.193 & 0.000 & 0.992 & 0.998 & 0.684 & 1.457 \\
Clinical stages & 0.623 & 0.226 & 7.586 & $0.006^{*}$ & 1.864 & 1.197 & 2.902 \\
Pathological grades & 0.499 & 0.208 & 5.763 & $0.016^{*}$ & 1.647 & 1.096 & 2.476 \\
WDR79 & 0.451 & 0.212 & 4.519 & $0.034^{*}$ & 1.570 & 1.036 & 2.379 \\
mtp53 & 0.325 & 0.196 & 2.746 & 0.097 & 1.384 & 0.942 & 2.032 \\
\hline
\end{tabular}

Abbreviation: LNM, lymph node metastasis; HR, hazard ratio; 95\% CI, 95\% confidence interval.

NOTE:* multivariate Cox's proportional hazard regression analysis. $P<0.05$ was regarded as statistically significant.

Besides Kaplan-Meier estimator, multivariate Cox proportional hazard regression analysis was also performed to further investigate whether these parameters, especially WDR79 and mtp53 expression, can act as the independent prognostic factors for overall survival status in NSCLC. Consistent with the existing view, gender, LNM status, clinical stages and pathological grades were independent risk factor for NSCLC patients. Notably, WDR79 positive expression was an independent risk factor for NSCLC patients $(P=0.034)$ (Table 3$)$. There were no prognostic effects detected in NSCLC patients including age, smoking status, histological type, treatment strategy and mtp53 expression (all $P>0.05$ ).

\section{Discussion}

WDR79, originally known as P53 antisense gene [12], not only maintains Cajal bodies ${ }^{[11,20,21]}$ and targets telomeres elongation via Cajal bodies[21], but also involves in DNA damage response [22, 23]. Previous studies have shown that WDR79 may play an important role in the progression and prognostication of numerous cancers, such as in breast cancer [24], head and neck carcinoma [15], rectal cancer [25], esophageal squamous cell carcinoma [26] and laryngeal squamous cell carcinoma [27]. In cancer, WDR79 facilitates the repair of DNA double-strand breaks and thus promotes cell survival [28]. Recently, researchers also found that WDR79 may involve in increased risk for ER negative breast cancer [24]. In NSCLC, WDR79 overexpression may involve in tumorigenesis, and in vitro study, Sun et al. found that WDR79 promoted NSCLC cell proliferation [14, 29,30]. It was reported that WDR79 colocalized and interacted with USP7, which reduced the ubiquitination of $\mathrm{Mdm} 2$ and p53, and thereby increased the stability and extended the half-life of the two proteins ${ }^{[29] .}$ 
We previously found that mtp53 is frequently overexpressed in human lung cancer. And mutant p53 cooperates with ETS2 to promote etoposide resistance in the NSCLC cell lines [31]. An immunohistochemical and nucleotide sequencing analysis indicated that IHC staining patterns of p53 can serve as a surrogate marker for TP53 mutations in ovarian carcinoma. In clinic, p53 staining via IHC can distinguish between wild-type and mutant p53 status. Immunohistochemical analysis can be used as a robust method for inferring the presence of a TP53 mutation in ovarian carcinomas [9]. Optimized p53 immunohistochemistry is an accurate predictor of TP53 mutation in ovarian carcinoma [19]. While NGS for TP53 gene is technically demanding and costly, this limits its application. It would be extremely rewarding to identify potential markers that are related to p53 and can be easily detected with conventional IHC method, which holds promise as prognostic factor or effective biomarkers.

In this study, WDR79 and mtp53 protein expression was detected in 289 cases of NSCLC tissues and 53 non-cancerous control lung tissues. WDR79 was located both in the cytoplasm and nucleus while mtp53 only located in the nucleus. However, they were both increased expression in NSCLC tissues. Then we further explored associations between expression of WDR79 and mtp53 and clinicopathological characteristics of NSCLC. We found that increased expression WDR79 was related with lymph node metastasis, and common expression of WDR79 and mtp53 proteins or WDR79 alone may indicate poor prognosis in the patients with surgically resected NSCLC. Furthermore, WDR79 expression was associated with expression of $\mathrm{mtp} 53$ protein in the NSCLC. With the Kaplan-Meier estimator and multivariate Cox's proportional hazard regression analysis, we found that WDR79 can be an independent poor prognosis marker in NSCLC and may be a tumor promoter. WDR79 as a poor prognosis biomarker could be well-detected by IHC, which is of great value in clinic application.

In summary, we first reported that positive expression of WDR79 protein was associated with TP53 mutation in the patients with surgically resected NSCLC, and positive expression of WDR79 might be regarded as one novel independent poor prognosis factor in patients with surgically resected NSCLC.

\section{Acknowledgements}

This work was supported by the National Natural Science Foundations of China (No: 81773218, 81472773, 81703009 and 81272566).

\section{Authors' contributions}

All authors contributed to the conception and design of the manuscript, collection and assembly of data, data analysis and interpretation, manuscript writing and final approval.

\section{Competing Interests}

The authors have declared that no competing interest exists.

\section{References}

1. Torre LA, Siegel RL, Ward EM, et al. Global Cancer Incidence and Mortality Rates and Trends--An Update. Cancer Epidemiol Biomarkers Prev. 2016; 25(1):16-27.

2. Hirsch FR, Scagliotti GV, Mulshine JL, et al. Lung cancer: current therapies and new targeted treatments. Lancet. 2017;389(10066):299-311

3. Siegel RL, Miller KD, Jemal A. Cancer statistics, 2017. CA Cancer J Clin. 2017;67(1):7-30

4. Hainaut P, Pfeifer GP. Somatic TP53 Mutations in the Era of Genome Sequencing. Cold Spring Harb Perspect Med. 2016; 6(11). pii: a026179

5. Goh AM, Coffill CR, Lane DP. The role of mutant p53 in human cancer. J Pathol. 2011;223(2):116-26

6. Takahashi T, Nau MM, Chiba I, et al. p53: a frequent target for genetic abnormalities in lung cancer. Science. 1989; 246(4929):491-494.

7. Hainaut $\mathrm{P}$, Soussi T, Shomer B, et al. Database of p53 gene somatic mutations in human tumors and cell lines: updated compilation and future prospects. Nucleic Acids Res. 1997; 25(1):151-157.

8. Mogi A, Kuwano H. TP53 mutations in nonsmall cell lung cancer. J Biomed Biotechnol. 2011; 2011:583929.

9. Yemelyanova A, Vang R, Kshirsagar M, et al. Immunohistochemical staining patterns of p53 can serve as a surrogate marker for TP53 mutations in ovarian carcinoma: an immunohistochemical and nucleotide sequencing analysis. Mod Pathol. 2011; 24(9):1248-1253.

10. Steels E, Paesmans M, Berghmans T, et al. Role of $\mathrm{p} 53$ as a prognostic factor for survival in lung cancer: a systematic review of the literature with a meta-analysis. Eur Respir J. 2001; 18(4):705-719.

11. Tycowski KT, Shu MD, Kukoyi A, et al. A conserved WD40 protein binds the Cajal body localization signal of scaRNP particles. Mol Cell. 2009; 34(1):47-57.

12. Mahmoudi S, Henriksson S, Corcoran M, et al. Wrap53, a natural p53 antisense transcript required for p53 induction upon DNA damage. Mol Cell. 2009; 33(4):462-71

13. Polson A, Durrett E, Reisman D. A bidirectional promoter reporter vector for the analysis of the p53/WDR79 dual regulatory element. Plasmid. 2011; 66(3):169-179.

14. Sun Y, Yang C, Chen J, et al. Overexpression of WDR79 in non-small cell lung cancer is linked to tumour progression. J Cell Mol Med. 2016; 20(4):698-709.

15. Sun $\mathrm{CK}$, Luo XB, Gou YP, et al. TCAB1: a potential target for diagnosis and therapy of head and neck carcinomas. Mol Cancer. 2014; 13:180.

16. Wen Q, Wang W, Luo J, et al. CGP57380 enhances efficacy of RAD001 in non-small cell lung cancer through abrogating mTOR inhibition-induced phosphorylation of eIF4E and activating mitochondrial apoptotic pathway. Oncotarget. 2016; 7(19):27787-801.

17. Wen $\mathrm{Q}$, Wang W, Chu S, et al. Flot-2 Expression Correlates with EGFR Levels and Poor Prognosis in Surgically Resected Non-Small Cell Lung Cancer. PLoS One. 2015; 10(7):e0132190.

18. Xu L, Jiang $\mathrm{Y}$, Zheng J, et al. Aberrant expression of $\beta$-catenin and E-cadherin is correlated with poor prognosis of nasopharyngeal cancer. Hum Pathol. 2013; 44(7):1357-1364

19. Köbel M, Piskorz AM, Lee S, et al. Optimized p53 immunohistochemistry is an accurate predictor of TP53 mutation in ovarian carcinoma. J Pathol Clin Res. 2016;2(4):247-258.

20. Mahmoudi S, Henriksson S, Weibrecht I, et al. WRAP53 is essential for Cajal body formation and for targeting the survival of motor neuron complex to Cajal bodies. PLoS Biol. 2010; 8(11):e1000521.

21. Venteicher AS, Abreu EB, Meng Z, et al. A human telomerase holoenzyme protein required for Cajal body localization and telomere synthesis. Science. 2009; 323(5914):644-648.

22. Henriksson S, Rassoolzadeh H, Hedström E, et al. The scaffold protein WRAP $53 \beta$ orchestrates the ubiquitin response critical for DNA double-strand break repair. Genes Dev. 2014; 28(24):2726-2738.

23. Coucoravas C, Dhanjal S, Henriksson S, et al. Phosphorylation of the Cajal body protein WRAP53 $\beta$ by ATM promotes its involvement in the DNA damage response. RNA Biol. 2017; 14(6):804-813

24. Garcia-Closas M, Kristensen V, Langerød A, et al. Common genetic variation in TP53 and its flanking genes, WDR79 and ATP1B2, and susceptibility to breast cancer. Int J Cancer. 2007; 121(11):2532-2538. 
25. Zhang H, Wang DW, Adell G, et al. WRAP53 is an independent prognostic factor in rectal cancer- a study of Swedish clinical trial of preoperative radiotherapy in rectal cancer patients. BMC Cancer. 2012; 12:294.

26. Rao X, Huang D, Sui X, et al. Overexpression of WRAP53 is associated with development and progression of esophageal squamous cell carcinoma. PLoS One. 2014; 9(3):e91670.

27. Qiu H, Zhao DY, Yuan LM, et al. Regulatory effects of WRAP53 on radiosensitivity of laryngeal squamous cell carcinoma cells. Asian Pac J Cancer Prev. 2015; 16(7):2975-2979.

28. Rassoolzadeh H, Böhm S, Hedström E, et al. Overexpression of the scaffold WD40 protein WRAP53 $\beta$ enhances the repair of and cell survival from DNA double-strand breaks. Cell Death Dis. 2016; 7:e2267.

29. Sun Y, Cao L, Sheng X, et al. WDR79 promotes the proliferation of non-small cell lung cancer cells via USP7-mediated regulation of the Mdm2-p53 pathway. Cell Death Dis. 2017; 8(4):e2743.

30. Chen J, Sheng X, Ma H, et al. WDR79 mediates the proliferation of non-small cell lung cancer cells by regulating the stability of UHRF1. J Cell Mol Med. 2018; 22(5):2856-2864.

31. Do PM, Varanasi L, Fan S, et al. Mutant $\mathrm{p} 53$ cooperates with ETS2 to promote etoposide resistance. Genes Dev. 2012; 26(8):830-845. 